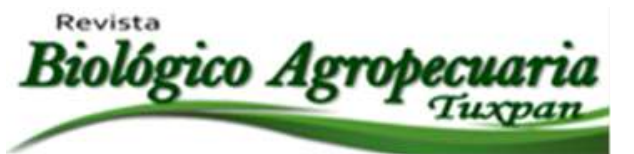

\title{
Uso de módulos en traspatio para cultivo de hortalizas con mayor demanda en la zona de Tuxpan, Veracruz
}

Use of backyard modules for growing vegetables with greater demand in the area of Tuxpan, Veracruz

Mendo Muñoz Rubén De La Paz ${ }^{1 凶}$, González Salas Miguel A. ', Sánchez Sotelo Edith Celeste ${ }^{1}$, Gutiérrez Vivanco Jordán ${ }^{1}$, Martínez Sánchez Cesar E. ${ }^{1}$

${ }^{1}$ Facultad de Ciencias Biológico Agropecuarias, Universidad Veracruzana. Campus Poza Rica-Tuxpan Carretera Tuxpan-Tampico Kilómetro 7.5, Universitaria, 92870 Tuxpan, Veracruz.

${ }^{\bowtie}$ Autor para correspondencia: rubmunoz@uv.mx

Recibido: $15 / 09 / 2018$

Aceptado: 15/11/2018

\section{RESUMEN}

En el mundo se han desarrollado en el sector agropecuario tecnologías innovadoras que como principal meta aumentar la calidad de los productos y aumentar el volumen, actualmente uno de los objetivos más importantes, es el cuidado del medio ambiente y el consumo de alimentos sanos. La principal innovación de este proyecto son los cultivos hidropónicos en módulos para poder cultivar hortalizas en espacios pequeños de las casas (traspatio). Estos cultivos tienen la ventaja de producir cosechas en un menor tiempo. Por lo anterior, se planeó implementar el uso de módulos en traspatio para cultivo de hortalizas con mayor demanda en la zona de Tuxpan, Veracruz. Se realizaron encuestas tomando en cuenta sólo a la población económicamente activa de un rango de edades de 18 a más de 40 años, obteniendo un tamaño de muestra de 382 personas a encuestar, el objetivo de las encuestas fue principalmente determinar el tipo de hortalizas que tienen mayor demanda y el tipo de módulos que los ciudadanos estarían dispuestos a adquirir para producir sus hortalizas para propio consumo y/o comercialización. Los resultados que se obtuvieron mostraron: que de las 382 personas encuestadas, se confirmó el consumo de hortalizas por 1135 personas más; los sitios preferidos para adquirir las hortalizas son las tiendas pequeñas locales y el mercado; las hortalizas que tienen mayor demanda son tomate, tomate verde, chile, cebolla, cilantro, calabacita, acelgas, espinacas, lechuga, papa ajo, rábanos, zanahoria, brócoli, frijol, coliflor, epazote y berenjena; las principales causas de consumo de hortalizas son por salud y nutrición; el $47 \%$ de la población tiene noción de técnicas de hidroponía, afirma que consumirían productos cultivados bajo dicha técnica, les gustaría tener su propio módulo hidropónico y le gustaría adquirir el paquete tecnológico; y finalmente, a 336 personas encuestadas estarían interesado en adquirir algún módulo hidropónico, de los cuales sólo 186 están dispuestos a pagar un valor de \$1000.00 a \$3000.00 pesos.

Palabras clave: Hidroponía, propio consumo, comercialización, paquetes. 


\begin{abstract}
In the world, innovative technologies have been developed in the agricultural sector, which as a main goal to increase the quality of products and increase volume, currently one of the most important objectives, is the care of the environment and the consumption of healthy food. The main innovation of this project are the hydroponic crops in modules to be able to grow vegetables in small spaces of the houses (backyard). These crops have the advantage of producing crops in a shorter time. Therefore, it was planned to implement the use of backyard modules for growing vegetables with greater demand in the area of Tuxpan, Veracruz. Surveys were carried out taking into account only the economically active population of a range of ages from 18 to over 40 years, obtaining a sample size of 382 people to survey, the objective of the surveys was mainly to determine the type of vegetables that have greater demand and the type of modules that citizens would be willing to acquire to produce their vegetables for own consumption and / or commercialization. The results that were obtained showed that of the 382 people surveyed, the consumption of vegetables was confirmed by 1135 more people; the preferred sites to acquire the vegetables are the small local shops and the market; the vegetables that are most in demand are tomato, green tomato, chile, onion, coriander, zucchini, chard, spinach, lettuce, garlic potato, radishes, carrots, broccoli, beans, cauliflower, epazote and aubergine; The main causes of vegetable consumption are health and nutrition; $47 \%$ of the population has a notion of hydroponics techniques, affirms that they would consume products grown under said technique, would like to have their own hydroponic module and would like to acquire the technological package; and finally, 336 people surveyed would be interested in acquiring a hydroponic module, of which only 186 are willing to pay a value of $\$ 1000.00$ to $\$ 3000.00$ pesos.
\end{abstract}

Keywords: Hydroponics, own consumption, marketing, packages.

\section{INTRODUCCIÓN}

Debido a los cambios climáticos, el desgaste del suelo y la falta de espacio en zonas urbanas para cultivar y producir vegetales, se ha vuelto prioridad de México y el mundo la creación de tecnologías para la producción que se ajusten a las situaciones actuales del clima de los diferentes países con el objetivo de aumentar la calidad de los productos y aumentar el volumen de éstos (Promuevehidroponia, 2014). Dos de las principales tecnologías que sobresalen por su alto grado de control y manejo de los factores que limitan la producción son los invernaderos y la hidroponía, sin embargo tienen la desventaja de requerir una inversión inicial muy elevada por lo que se recomienda producir cultivos de alto valor comercial para elevar su rentabilidad (Rodríguez, 2005).

Las hortalizas son un producto de alta demanda, dada su importancia en la nutrición familiar por su gran cantidad de vitaminas, minerales y proteínas que aportan (FAO, 2011); lo que las vuelve ideales para ser cultivadas en módulos de cultivo, los cuales tienen como principal innovación, poder cultivar cualquier hortaliza en espacios muy reducidos de los hogares, sin importar las condiciones climáticas en las que se encuentre el entorno (TRAXCO, 2012; Beltrano y Giménez, 2015). Este tipo de cultivos tienen múltiples ventajas ya que, produce en menor tiempo en comparación a los cultivos convencionales, mayor producción (se puede duplicar o triplicar), sólo se usa sustratos o agua, 
los nutrimentos que necesitan se proporcionan por soluciones directo a la planta y se evitan la utilización de químicos que son nocivos para el ser humano (Beltrano y Giménez, 2015).

Por lo ya mencionado, el presente trabajo tuvo como objetivos, identificar las hortalizas de mayor demanda en el municipio de Tuxpan, Veracruz; y la creación de un módulo de cultivo caseros (de traspatio) para producirlas de forma agroecológica con el fin de mejorar la economía de quién las produce al emplearlas para autoconsumo o/y comercializarlas.

\section{MATERIALES Y MÉTODOS}

El estudio se llevó a cabo en el municipio de Tuxpan de Rodríguez cano Veracruz, el cual cuenta 360 localidades, de estas 3 son comunidades urbanas y los 357 restantes son rurales.

Las principales actividades económicas que tiene el municipio son agricultura, ganadería, pesca, así como turismo y comercio nacional e internacional. El municipio de Tuxpan cuenta con una superficie de 75,812.448 hectáreas, de las cuales sólo 58.339.104 hectáreas se utilizan para la siembra, principalmente de naranja, maíz, frijol y chile.

Para poder establecer las hortalizas con mayor demanda en la zona, y el diseño de módulos de cultivo que se propondrán, se realizaron encuestas tomando en cuenta sólo a la población económicamente activa de un rango de edades de 18 a más de 40 años, obteniendo un tamaño de muestra de 382 personas a encuestar.

\section{RESULTADOS Y DISCUSIÓN}

Los resultados más relevantes que se obtuvieron fueron los siguientes:

De las 382 personas encuestadas, se confirmó el consumo de hortalizas por 1135 personas más. Los sitios preferidos para adquirir las hortalizas son las tiendas pequeñas locales y el mercado.

Cuadro 1. Consumo aproximado de la población encuestada

\begin{tabular}{|c|c|}
\hline HORTALIZA & CONSUMO POR SEMANA \\
\hline Tomate & 710.5 kilos \\
\hline Tomate verde & 518 kilos \\
\hline Chile & 358.5 kilos \\
\hline Cebolla & 468.5 kilos \\
\hline Cilantro & 294 rollos \\
\hline Calabacita & 426 kilos \\
\hline Acelgas & 355 rollos \\
\hline Espinacas & 156 rollos \\
\hline Lechuga & 452 piezas \\
\hline Papa & 656 kilos \\
\hline
\end{tabular}


Cuadro 2. Precio promedio de las hortalizas.

\begin{tabular}{|c|c|}
\hline HORTALIZA & PRECIO \\
\hline Tomate & $\$ 20$ \\
\hline Chile & $\$ 14$ \\
\hline Cebolla & $\$ 23$ \\
\hline Cilantro & $\$ 10$ \\
\hline Calabacita & $\$ 25$ \\
\hline Acelga & $\$ 7$ \\
\hline Espinaca & $\$ 13$ \\
\hline Lechuga & $\$ 18$ \\
\hline Papa & $\$ 19$ \\
\hline
\end{tabular}

-Las principales causas de consumo son por salud y nutrición.

-El 47\% de la población tiene noción de técnicas de hidroponía, afirma que consumirían productos cultivados bajo dicha técnica, les gustaría tener su propio módulo hidropónico y le gustaría adquirir el paquete tecnológico.

-Los encuestados sugieren sembrar tomate, chile, cebolla, cilantro, papa, lechuga, calabacitas, espinacas, ajo, rábanos, zanahoria, brócoli, frijol, coliflor, epazote y berenjena.

-336 personas estarían interesado en adquirir algún módulo hidropónico, de los cuales sólo
186 están dispuestos a pagar un valor de $\$ 1000.00$ a $\$ 3000.00$ pesos.

De acuerdo a la encuestas realizadas se determinó que existen diferentes hortalizas de importancia comercial, por lo que partiendo de esa información, se elaboraron cinco paquetes que incluyen de tres a cinco hortalizas. Los paquetes que tuvieron preferencia por los encuestados fueron dos paquetes con la demanda más alta, el paquete tres con $36 \%$ compuesto por los cultivos de chile, acelga, espinaca, lechuga y tomate, y el paquete cuatro con $30 \%$ compuesto por los cultivos de tomate, lechuga y espinaca. 


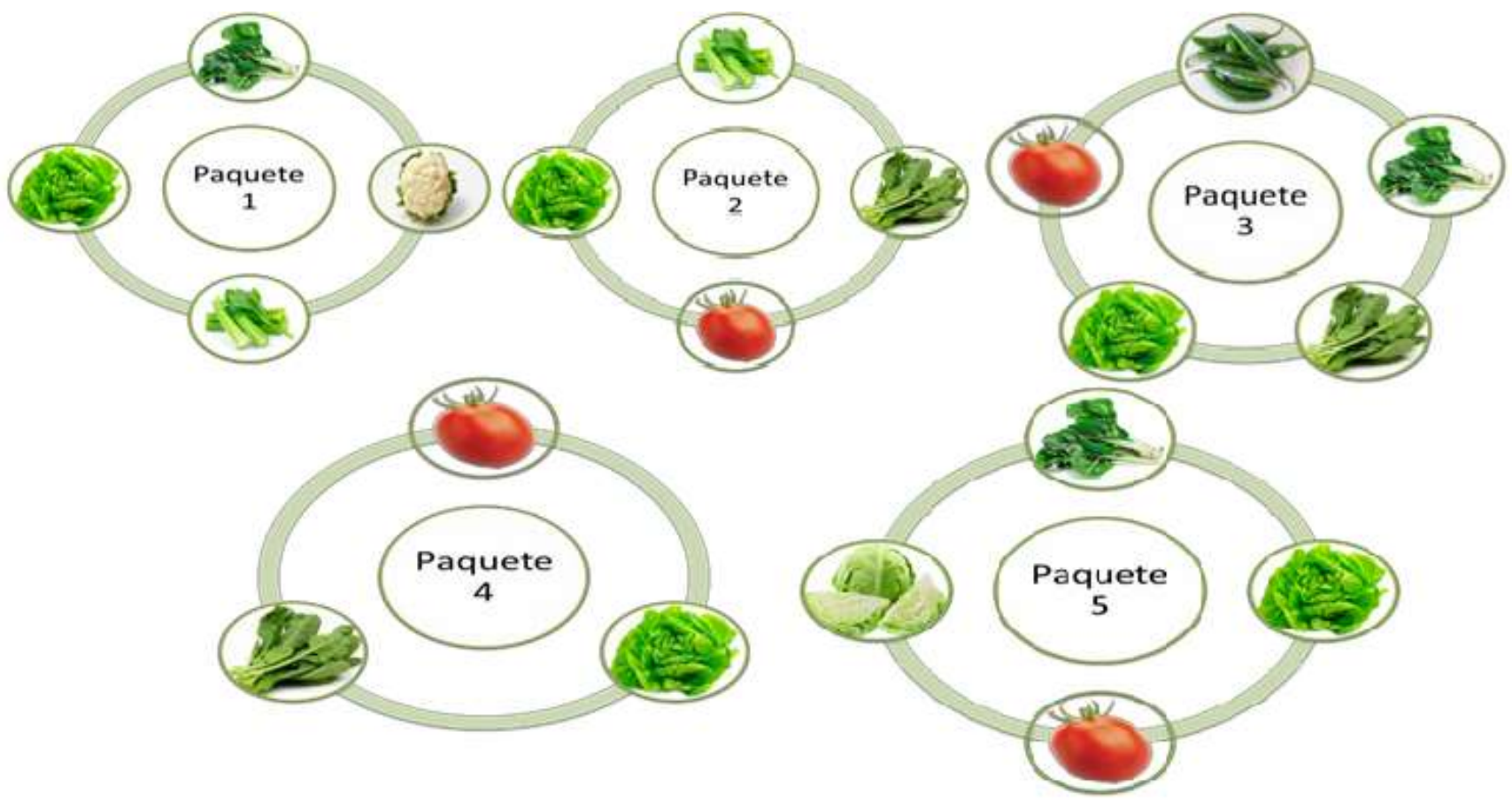

Figura 1. Paquetes sugeridos por la preferencia de la población encuestada.

A los encuestados se les explicó a grandes rasgos en qué consiste cada módulo hidropónico, y seleccionado por los interesados en implementarlo para la producción de hortalizas en traspatio, fue el módulo NFT, el cual consiste en una técnica utilizando una lámina de nutrientes, se adapta a lugares con poco espacio ya que este también puede hacerse por niveles y tiene una gran capacidad para plantas pequeñas y medianas. Se utilizan tubos PVC o Vinyl estos son tratados contra los rayos $\mathrm{UV}$, los tubos se perforan donde crecerán las plantas, el agua con los nutrientes se bombea desde un recipiente hacia los tubos y como si fuera un ciclo vuelve al recipiente principal. Así el agua que van por los tubos alimenta las plantas (Urveg, 2017).
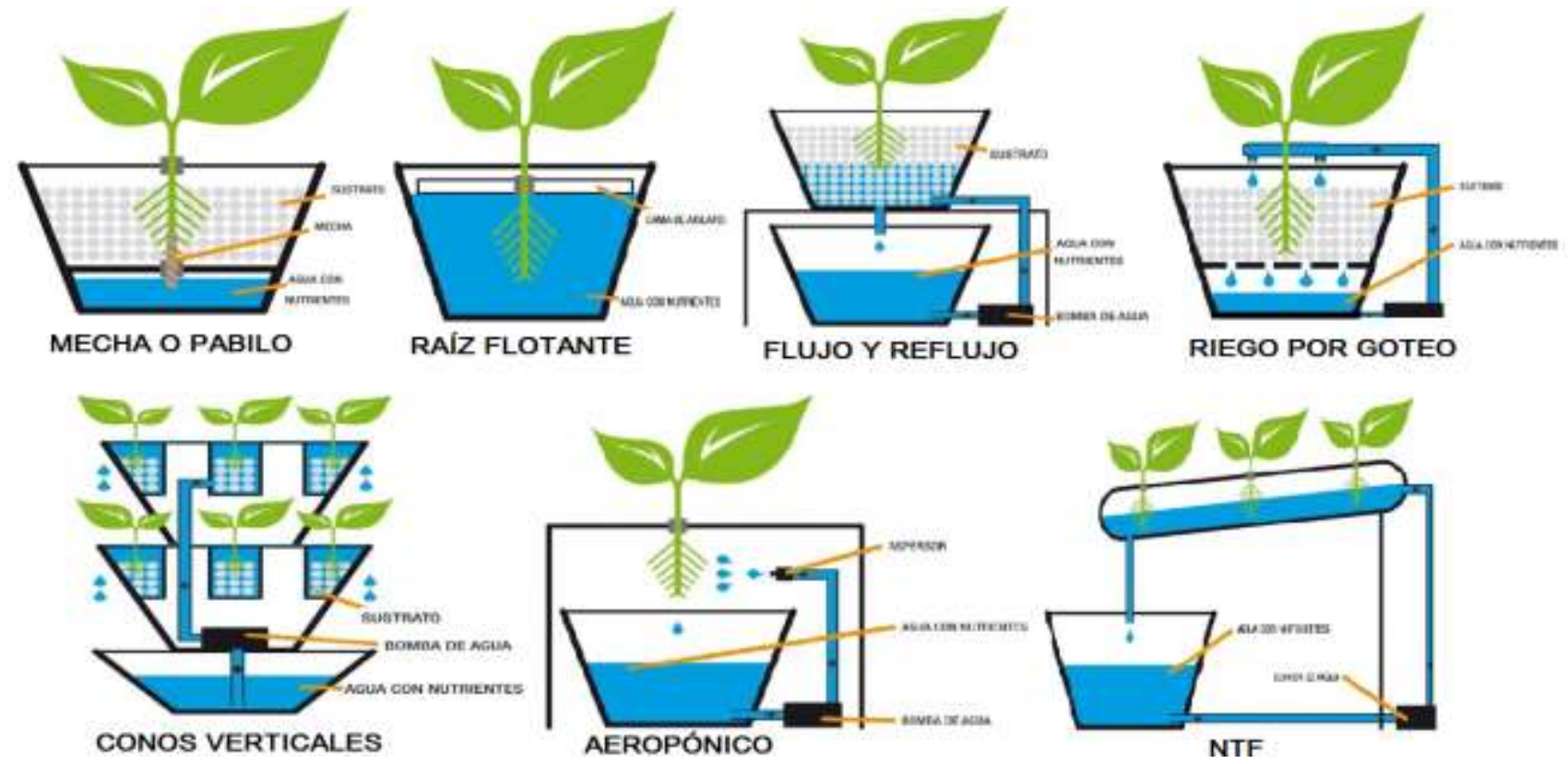

Figura 2. Tipos de módulos hidropónicos propuestos.

Revista Científica Biológico Agropecuaria Tuxpan 6 (2) 
Tomando en consideración los paquetes y el tipo de módulo elegido, se realizó la estimación de la producción de hortalizas por paquete, dicha estimación incluye el tiempo de los cultivos en semanas, el número de plantas que se van a cultivar en el módulo y la distancia que tendrán entre ellas. El rendimiento por cultivo en el módulo de que se va a manejar, la producción que se tiene por año de cada cultivo y el costo por cada $\mathrm{kg}$ y rollo de los cultivos. Así como el ahorro y los beneficios adicionales generados por ambos módulos.

\begin{tabular}{|c|c|c|c|c|c|c|c|}
\hline Paquete 3 & $\begin{array}{c}\text { Tiempo del } \\
\text { cultivo/Semanas }\end{array}$ & $\begin{array}{c}\text { No. Planta } \\
1.30 \mathrm{~m}\end{array}$ & $\begin{array}{c}\text { Rendimiento } \\
\text { estimado } \\
\text { NFT }\end{array}$ & $\begin{array}{r}\text { Siembras } \\
\text { /año }\end{array}$ & $\begin{array}{r}\text { Producción } \\
\text { /año }\end{array}$ & $\begin{array}{c}\text { Costo por } \\
\mathrm{Kg} / \mathrm{Pz}\end{array}$ & Total \\
\hline $\begin{array}{c}\text { Jitomate Inicia } \\
\text { Cosecha } 13 \text { Semanas }\end{array}$ & 20 & $\begin{array}{c}5 \\
\mathrm{c} / 25\end{array}$ & $30 \mathrm{~kg} /$ planta & 2 & $300 \mathrm{~kg}$ & $\$ 20$ & $\$ 6,000$ \\
\hline $\begin{array}{c}\text { Lechuga Semanas } \\
12 \text {-16 Cosecha } \\
\text { hoja }\end{array}$ & 31 floración & $\begin{array}{c}5 \\
c / 25\end{array}$ & $1000 \mathrm{gr} /$ planta & 4 & $20 \mathrm{~kg}$ & $\$ 18$ & $\$ 360$ \\
\hline $\begin{array}{c}\text { Espinacas } \\
\text { Cosecha 8-10 } \\
\text { Semanas }\end{array}$ & 14 & $\begin{array}{c}5 \\
\mathrm{c} / 8 \mathrm{~cm}\end{array}$ & 200gr/planta & 4 & $\begin{array}{c}4 \mathrm{~kg} \\
40 \text { rollos } \\
\text { de } 100 \mathrm{gr}\end{array}$ & $\$ 13$ & $\$ 520$ \\
\hline $\begin{array}{c}\text { Acelgas } \\
\text { Cosecha 8- } 10 \\
\text { Semanas }\end{array}$ & & $\begin{array}{c}3 \\
\mathrm{c} / 25\end{array}$ & $2 \mathrm{Kg} /$ planta & 4 & $\begin{array}{c}24 \mathrm{~kg} \\
80 \text { rollos } \\
\text { de } 300 \mathrm{gr}\end{array}$ & $\$ 7$ & $\$ 560$ \\
\hline $\begin{array}{c}\text { Chile Serrano } \\
\text { Cosecha Semanas 16- } \\
20\end{array}$ & 20 & $\begin{array}{c}5 \\
c / 25\end{array}$ & $2.5 \mathrm{~kg} / \mathrm{planta}$ & 2 & $25 \mathrm{~kg}$ & $\$ 14$ & $\$ 350$ \\
\hline Total & & 23 & & & $374 \mathrm{~kg}$ & & $\$ 7,790$ \\
\hline
\end{tabular}

Figura 3. Producción del módulo de hidroponía NFT para el paquete 3. 
Mendo et al., 2018

\begin{tabular}{|c|c|c|c|c|c|c|c|}
\hline Paquete 4 & $\begin{array}{c}\text { Tiempo del } \\
\text { cultivo/Semanas }\end{array}$ & $\begin{array}{r}\text { No. Planta } \\
1.30 \mathrm{~m}\end{array}$ & $\begin{array}{c}\text { Rendimiento } \\
\text { estimado } \\
\text { NFT }\end{array}$ & $\begin{array}{c}\text { Siembras } \\
\text { / año }\end{array}$ & $\begin{array}{l}\text { Producción } \\
\text { / año }\end{array}$ & $\begin{array}{c}\text { Costo por } \\
\mathrm{Kg} / \mathrm{Pz}\end{array}$ & Total \\
\hline $\begin{array}{c}\text { Jitomate Inicia } \\
\text { Cosecha } 13 \\
\text { Semanas }\end{array}$ & 20 & $5 \mathrm{c} / 25$ & $30 \mathrm{~kg} /$ planta & 2 & $300 \mathrm{~kg}$ & $\$ 20$ & $\$ 6,000$ \\
\hline $\begin{array}{c}\text { Lechuga } \\
\text { Semanas } \\
12-6 \\
\text { Cosecha hoja }\end{array}$ & 31 floración & $10 \mathrm{c} / 25$ & $1000 \mathrm{gr} /$ planta & 4 & $40 \mathrm{~kg}$ & $\$ 18$ & $\$ 720$ \\
\hline $\begin{array}{c}\text { Espinacas } \\
\text { Cosecha 8-10 } \\
\text { Semanas }\end{array}$ & 14 & $15 \mathrm{c} / 8 \mathrm{~cm}$ & 200gr/planta & 4 & $\begin{array}{r}12 \mathrm{~kg} \\
120 \text { rollos de } \\
100 \mathrm{gr}\end{array}$ & $\$ 13$ & $\$ 1,560$ \\
\hline Total & & 25 & & & $352 \mathrm{~kg}$ & & $\$ 8,280$ \\
\hline
\end{tabular}

Figura 4. Producción del módulo de hidroponía NFT para el paquete 4.

\begin{tabular}{|c|c|c|c|c|c|c|c|c|}
\hline & $\begin{array}{c}\text { Producción/ } \\
\text { Año }\end{array}$ & \begin{tabular}{|l} 
Valor \\
Producción \\
\$/año
\end{tabular} & $\begin{array}{c}\text { Consume/ } \\
\text { Año }\end{array}$ & $\begin{array}{r}\text { Depreciación } \\
\text { Módulo }\end{array}$ & $\begin{array}{c}\text { Costo en el } \\
\text { Mercado } \\
\text { \$/año }\end{array}$ & $\begin{array}{r}\text { Costo de } \\
\text { Producción }\end{array}$ & Ahorro & $\begin{array}{c}\text { Beneficio } \\
\text { Adicional } \\
\text { \$/año }\end{array}$ \\
\hline $\begin{array}{c}\text { Paquete } \\
3\end{array}$ & $374 \mathrm{~kg}$ & 7,790 & $240 \mathrm{~kg}$ & $\$ 190$ & 3,456 & $\begin{array}{c}\$ 3,646 \\
\$ 15.19 / \mathrm{kg}\end{array}$ & $\begin{array}{c}240 \mathrm{~kg} / 15.19 \\
\$ 3,645\end{array}$ & 4,145 \\
\hline $\begin{array}{c}\text { Paquete } \\
4\end{array}$ & $352 \mathrm{~kg}$ & 8,280 & $144 \mathrm{~kg}$ & $\$ 190$ & 2,448 & $\begin{array}{c}\$ 2,638 \\
\$ 18.31 / \mathrm{kg}\end{array}$ & $\begin{array}{c}144 \mathrm{~kg} / 18.31 \\
\$ 2,636\end{array}$ & 5,644 \\
\hline
\end{tabular}

Figura 5. Ahorro y beneficios adicionales generados por los módulos.

\section{CONCLUSIÓN}

Las encuestas realizadas al tamaño de muestra de 382 personas encuestas indicó que el $47 \%$ de la población económicamente activa encuestada manifestó tener conocimiento sobre la existencia de los cultivos de hidroponía y manifestaron tener interés en adquirir un módulo para producir sus propios cultivos sanos con un valor aproximado de $\$ 1000.00$ a $\$ 3000.00$ pesos. Se determinaron los dos paquetes con la demanda más alta, el 3 con $36 \%$ compuesto por los cultivos de chile, acelga, espinaca, lechuga y tomate, y 4 con $30 \%$ compuesto por los cultivos de tomate, lechuga y espinaca. Y el módulo hidropónico seleccionado fue el modelo NFT.
De acuerdo con las estimaciones de producción, se prevé un excelente panorama para cubrir la demanda de hortalizas de la familia por módulo de $374 \mathrm{Kg}$ por año del paquete tres generando un ahorro de $\$ 3,456.00$, teniendo en cuenta que también genera un beneficio de $\$ 4,145.00$. El paquete cuatro tendrá una producción de hortalizas de $352 \mathrm{Kg}$ con lo que genera un ahorro de $\$ 2,636.00$ con un beneficio de $\$ 5,280.00$.

\section{LITERATURA CITADA}

Beltrano, J., y Giménez, D. O. (2015). Cultivo en hidroponía. Recuperado a partir de https://doi.org/10.35537/10915/46752 
FAO: Food and Agriculture Organization. (2011). Producción de Hortalizas. Recuperado a partir de http://www.fao.org/3/a-as972s.pdf

Promuevehidroponia. (2014). Hidroponía: una opción para contrarrestar el calentamiento global - hidroponia.mx. Recuperado el 16 de enero de 2018, a partir de http://hidroponia.mx/hidroponia-unaopcion-para-contrarrestar-elcalentamiento-global/

Rodríguez, M. E. (2005). Producción de hortalizas bajo sistemas hidropónicos, técnica de la película de nutriente (NFT) y cama de agua. Universidad Tecnológica de la Mixteca, Oaxaca. Recuperado a partir de

http://jupiter.utm.mx/ -tesis_dig/9735.pdf

TRAXCO. (2012). La contaminación del aire y la agricultura - La lluvia ácida. Recuperado el 16 de enero de 2018, a partir de https://www.traxco.es/blog/noticiasagricolas/la-contaminacion-del-aireafecta-a-la-agricultura.

Urveg. (2017). Principales características de los sustratos hidropónicos. Recuperado el 28 de octubre de 2017, a partir de http://hidroponia.mx/principalescaracteristicas-de-los-sustratoshidroponicos/

Copyright (c) 2018 Rubén de la Paz Mendo Muñoz, Miguel A. G onzález Salas, Edith Celeste Sánchez Sotelo, Jordán Gutiérrez Vivanco y César E. Martinez Sánchez

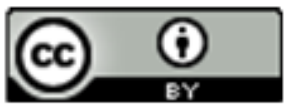

E ste tex to está protegido por una licencia Creative Commons 4.0

Usted es libre para Compartir — copiar y redistribuir el m aterial en cualquier medio o formato-y Adaptar el documento —remezclar, transformar y crear a partir del material- para cualquier propósito, incluso para fines com erciales, siempre que cumpla la condición de:

Atribución: Usted debe dar crédito a la obra original de manera adecuada, proporcionar un enlace a la licencia, e indicar si se han realizado cambios. Puede hacerlo en cualquier forma razonable, pero no de forma tal que sugiera que tiene el apoyo del licenciante o lo recibe por el uso que hace de la obra.

Resumendelicencia - Textocompletodelalicencia 